\title{
The eduction of structures from flow imagery using wavelets Part I. The mixing layer
}

\author{
S. V. Kailas, R. Narasimha
}

\begin{abstract}
Using two-dimensional Mexican hat wavelets, digitized imagery from the flow visualization pictures of a mixing layer by Brown and Roshko have been analysed. The objective of the present study is to "calibrate" the proposed wavelet technique for educing structures in a flow whose large-scale organization is well established. It is found that, with appropriate thresholds on wavelet-transform coefficients, considerable insight into the structure of the flow at different scales can be obtained. In particular, the analysis reveals that the small-scale structure within the large-scale vortices is approximately homogeneous, and has characteristic scales that do not vary significantly with downstream distance.
\end{abstract}

\section{1}

\section{Introduction}

The first dramatic evidence of coherent structures in a fully developed turbulent flow came from the pioneering studies of Roshko and his co-workers (e.g. Brown and Roshko 1974; Roshko 1976) in mixing layers. Along with the studies of Kline et al. (1967) in a boundary layer, this body of work established that turbulence, despite its apparent disorder, possesses a considerable degree of order, and may exhibit an astonishing variety of coherent motions (see e.g. Robinson 1991 for a "taxonomy" of these motions in a turbulent boundary layer). Inspite of the extensive work on coherent structures in a variety of flows (reviewed by Hussian (1986) and Fiedler (1988)), inferring the nature and characteristics

Received: 5 March 1998/Accepted: 28 October 1998

S. V. Kailas

Centre for Civil Aircraft Design and Development

National Aerospace Laboratories, Bangalore 560 012, India

R. Narasimha

Fluid Dynamics Unit, Jawaharlal Nehru Centre for Advanced Scientific Research

Bangalore 560 064, India

Correspondence to: R. Narasimha

The computation of the wavelet transforms was accomplished using NALLETS ver 3.0, a software package in $\mathrm{VC}++$ developed at the National Aerospace Laboratories, Bangalore, India for analysis of complex turbulence data. The authors wish to thank Roopa V. Pai for her cheerful help in carrying out the computations and generating the graphics. The authors also acknowledge a helpful comment by an anonymous referee. of such structures in the presence of considerable disorder remains a difficult problem.

Much early work was done with straight-forward flow visualization. Although obtaining quantitative information from flow visualisation studies has often been a laborious, imprecise and ambiguous exercise, direct visualisation by shadowgraph, schlieren, interferometry, molecular or particle scattering or fluorescence is still a powerful technique in determining the gross features of large-scale structure in flows where it is clearly visible. In other cases (e.g. jets), our understanding of coherent motions in the fully developed region is less adequate. Laser sheet visualizations do not reveal order as clearly in jets as in mixing layers. Two-point space-time correlations of longitudinal velocity fluctuations (Tso and Hussain 1989) have been more successful, but such methods may under-estimate the degree of order present because of the averaging process inherent in the correlation technique, especially as there is considerable jitter in the structures, and their spatial scales and life-time are both highly variable (e.g. see the statistics of the mixing layer provided by Bernal 1988). If there are several types of structure of different scales, such techniques cannot reveal whether their occurrence is simultaneous or not.

With the advent of digital imagery, sophisticated measurement techniques (particle and holographic imaging velocimetry) and high-resolution direct numerical simulations, some sort of processing is often undertaken to obtain more or less quantitative information. Visual processing has in general been addressed by thresholding and averaging techniques; e.g. Cutler and Johnson (1997) have recently used thresholding on laser-illuminated digitised images of a plume to study the nature of intermittency in a supersonic flow with injection. (Such techniques are canonical even in the image-processing community.) With such methods the precise technique used may differ from experiment to experiment, sometimes even from image to image. An objective, easily applicable and universally understood method would therefore be most useful.

We explore here the idea that the wavelet transform may provide a logical framework for image processing that can be universally applied, and with some familiarity and calibration can provide less ambiguous interpretations. Thus the present work has two objectives, first to describe the use of the wavelet transform technique on flow visualisation pictures where the existence of large-scale coherent structures is unambiguous, and secondly to study the small-scale structure of the flow. We shall consider more complex flows in a future publication. 
2

\section{Data analysed}

The data analysed are two pictures kindly supplied by Prof. A. Roshko, respectively, of laminar and turbulent mixing layers obtained using schlieren photography. A plane mixing layer is selected for this first study as the coherent structure here is unambiguously clear.

The experimental set-up is described in detail in Brown and Roshko (1974). In these experiments the upper stream is helium at a free stream velocity $U_{1}=10 \mathrm{~m} / \mathrm{s}$ while the lower is nitrogen at velocity $U_{2}=3.8 \mathrm{~m} / \mathrm{s}$. The pressure was 8 bar in the turbulent flow (with Reynolds number $R e=3 \times 10^{6}$ based on the free stream velocity and kinematic viscosity of the fluid on the faster side and the width of the picture), and 2 bar in the laminar mixing layer $\left(R e=0.3 \times 10^{5}\right)$. The photographs are first recorded on video tape and the video signal is fed to an image digitizer. Digitisation is accomplished using a Data Translation (USA) frame grabber board DT2871 (along with a PAL to RGB converter board DT2869) placed inside a PC 486. The pictures are digitised with $500 \times 130$ (laminar) and $500 \times 151$ (turbulent) pixel resolution and 8 bit accuracy with 256 grey levels of image intensity.

\section{3}

\section{The wavelet transform}

The wavelet transform $\hat{f}(\mathbf{x}, a)$ of a function $f(\mathbf{x})$ may be defined as the convolution product of a scaled and shifted mother wavelet $\psi(\mathbf{x})$ with the function to be analysed:

$\hat{f}(\mathbf{x}, a)=\int f\left(\mathbf{x}^{\prime}\right) \psi\left(\frac{\mathbf{x}-\mathbf{x}^{\prime}}{a}\right) \mathrm{d} \mathbf{x}^{\prime}$

where $a$ is the scale parameter and $\mathbf{x}^{\prime}$ is the shift parameter. Detailed reviews of wavelet transform principles and techniques are available in Strang (1989) or Meyer (1993). Briefly, the mother wavelet must be a rapidly decaying function of its argument (i.e. must have compact support), have a zero mean to be "admissible", and be normalized suitably, usually to ensure invertibility and preservation of the $L^{2}$ norm. Several such functions exist in the wavelet literature. An invertible, orthonormal transform yields an "optimal" wavelet representation of $f(\mathbf{x})$ useful for such purposes as signal compression. For the present work, we use the continuous version of the transform which is richly redundant and hence facilitates analysis, in particular, because of the freedom it provides on the choice of wavelet scale. The transform is particularly useful because of its ability to identify localized regions of energy concentration. This property is its major advantage over conventional Fourier-type analysis, which provides only a global distribution of energy. In the case of a 2D image this implies the possibility of detecting regions of high activity at different physical scales.

The transform has been earlier used to study coherent structures in turbulence. Thus Farge (1992) reconstructed scale-specific regions of high vorticity concentration in direct numerical simulations by inverting the transform, and used it to study the dynamics of these structures. In the present case, we use the transform solely as a detector of structures at various spatial scales. For this purpose we specifically choose the two-dimensional Mexican hat wavelet,

$\psi(\mathbf{x})=\left(2-x^{2}+y^{2}\right) \exp -\left(x^{2}+y^{2}\right) / 2$ where $\mathbf{x} \equiv(x, y)$ is a two-dimensional position vector in the plane of the image being analysed, with $x$ along the centre-line of the mixing layer and $y$ normal to it. The zero-crossings of this transform have been shown to be particularly useful in detecting regions of sharp gradients in an image (Marr 1982). Such gradients may assist in identifying the interfaces between coherent and non-coherent motion.

For better appreciation of the physical scales of the structures detected, we shall in the present study replace the scale $a$ of the wavelet transform by $a^{\prime}$, the ratio of the wavelet scale to the length $L$ of the image along the $x$-axis.

4

\section{Results}

\section{1}

\section{The laminar mixing layer}

Two grey scale images, respectively of a laminar and a fully turbulent mixing layer, are shown in the top left panels of

Fig. 1 and 2. The grey scales are arranged linearly between the lowest and highest intensity values. It is clear that the large coherent structures so easily seen in the laminar case are also present in the turbulent mixing layer, albeit "super-imposed" on a "background" of fine scale turbulence (Roshko 1976). What is immediately obvious form these pictures is that there is no ambiguity in inferring the existence of coherent structures.

Figure 1 also shows the wavelet transform of the laminar layer at six dyadically increasing scales starting from $a^{\prime}=0.02$. Again the grey scales are arranged linearly with intensity; however white, centred around zero, demarcates the positive from the negative coefficients. Since the wavelet transform at the smallest scales is sensitive to the very fine scale noise occurring on an otherwise smooth background, it was necessary to remove the (almost unnoticeable) digitisation noise from the background ambient flow or non-mixing-layer region. This helps to prevent distraction from the main results. Initially, elaborate quantitative methods were tried to remove this noise, including the identification of thresholds from probability distributions of pixel intensity, edge detection by short-time variances and various refinements thereof. However, a simple definition of the edge by eye, leveling all pixels above and below the upper and lower edges (the "background") to a constant value and careful thresholding of the wavelet transform of the image at the smallest scale (see details below), proved most effective. The results are those displayed in Fig. 1.

The raw image was first carefully trimmed to retain the mixing layer and the background was uniformly set to the average pixel intensity of the edge of the mixing layer bordering the background. Thus the upper background was set to the average intensity of the upper edge and similarly the lower background. The resultant raw image is shown in Fig. 1. There was virtually no discernible difference between this version and the original image, except that the small scale background noise was completely eliminated. Two additional refinements were found to be necessary. There is a small amount of unmixed background (ambient fluid) within the laminar mixing layer, and this resulted in retention of noise at the lowest scale $\left(a^{\prime}=0.02\right)$. This was removed by examining the 




0

144
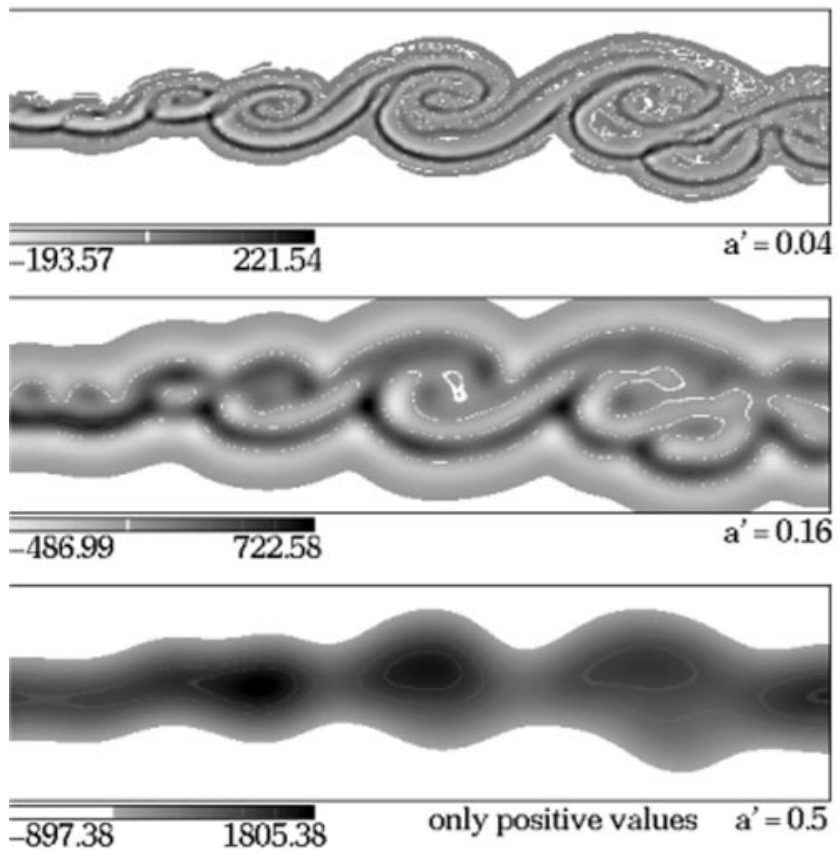

Fig. 1. Raw image of the laminar mixing layer $\left(U_{1}=10 \mathrm{~m} / \mathrm{s}\right.$, $U_{2}=3.78 \mathrm{~m} / \mathrm{s}$ and pressure $=2 \mathrm{bar}$ ), and wavelet transforms

wavelet transform coefficients at increasing thresholds both above and below zero. The wavelet-transformed image at $a^{\prime}=0.02$ shown in Fig. 1 displays the thresholded values which exhibit very little background noise, but retains all the essential features that were seen in the unprocessed transformed image. Since the fine scale noise is not picked up at larger scales (one of the reasons why the transform is used as a filter in signal processing applications, see e.g. Meyer 1993), the wavelet transforms at $a^{\prime}$ greater than 0.04 are displayed without any thresholding. At higher scales the best results were obtained when the pixels in the background outside the mixing layer were set to zero. The negative transforms (all occurring outside the mixing layer) are not displayed at $a^{\prime}=0.32$ and 0.5 for clarity, as they have no physical bearing on the flow features in any case.

Several features may be immediately noted in Fig. 1. At the smallest scale of $a^{\prime}=0.02$, the fine scale structure of the mixing layer stands out. The location of the regions or layers of maximum density gradient may be noted. These follow the regions of highest intensity in the raw image and are seen to be strikingly similar to it in nearly every detail. The wavelet transform therefore unambiguously picks out the finest regions of interest at the smallest scales. The analogy of the mixing layer and breaking rollers on a beach (Roshko 1976) is seen all the way up to $a^{\prime}=0.16$. The wavelet transform at $a^{\prime}=0.32$ actually shows the first three vortices closest to the


at six different scales

splitter plate as a continuous wave, while the fifth and sixth vortices from the splitter plate are seen to exhibit roller characteristics.

Also, as we go higher in scale, the regions of stronger and weaker gradients at each scale can be identified, e.g. at $a^{\prime}=0.08$ and 0.16 , the density gradients at the bottom are seen to be stronger (more nearly continuous and uniformly intense) than those at the top (less intense and discontinuous). Again, the curling up of the fluid layers in the core of the vortices, so clear at the lower scales, is seen to be replaced by fragmented, separate regions of varying intensity and size at higher scales. And the smallest two vortices (just off the splitter plate), distinctly seen as tiny rollers at $a^{\prime}=0.02$, appear as separate upper and lower layers at $a^{\prime}=0.04$ and 0.08 , while at $a^{\prime}=0.16$ the cores of these vortices are clearly demarcated as separate more intense regions.

At $a^{\prime}=0.32$, as already noted above, the first three vortices off the splitter plate are seen as a thick wavy sheet, the fourth is of just the right size to be resolved as an independent entity, while the fifth and sixth continue to show roller characteristics. At the highest scale $a^{\prime}=0.5$, the smallest three vortices are too small to be resolved separately, but the next three vortices are seen as distinct isolated entities. Thus, as we go up in scale the fine structure gets obliterated while the large-scale structure gets more clearly defined. Also, at the same wavelet scale, structures of different sizes (even if these have the same shape 

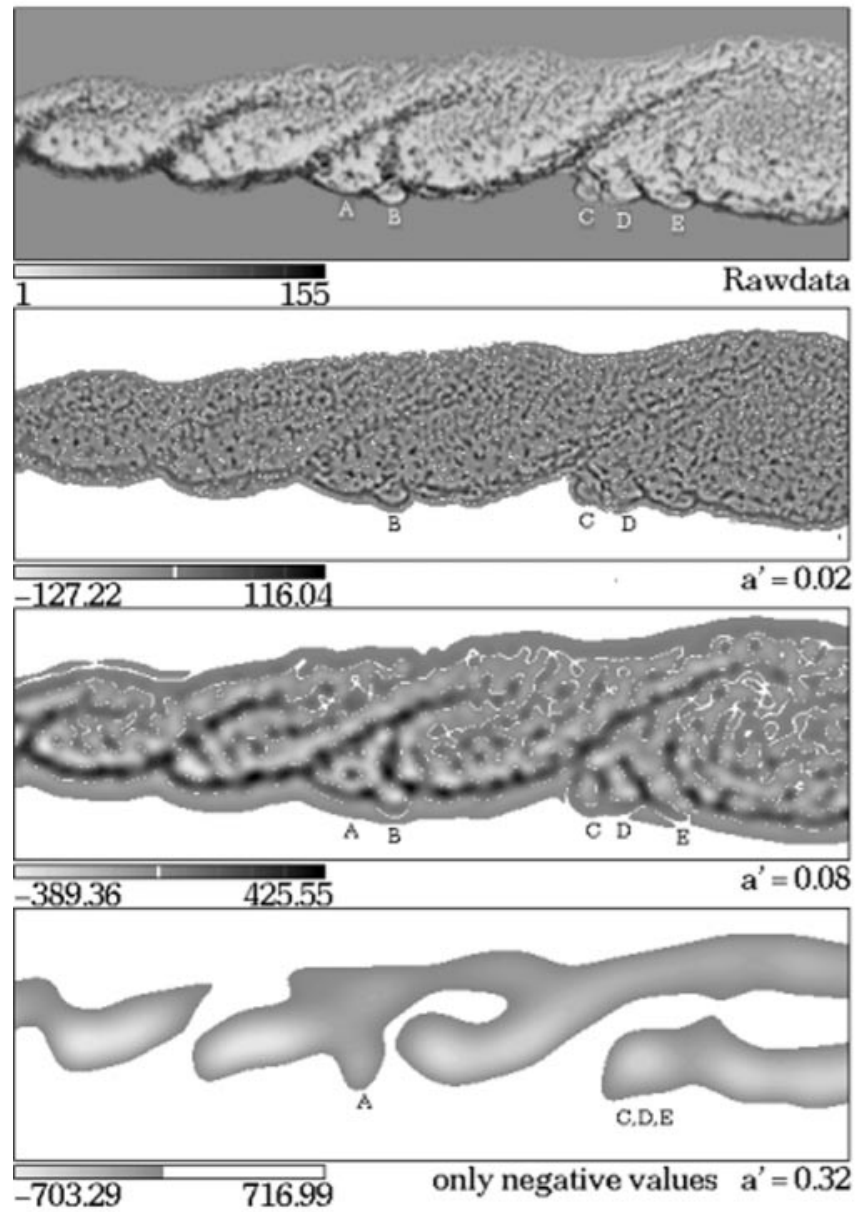

Fig. 2. Raw image of the turbulent mixing layer $\left(U_{1}=10 \mathrm{~m} / \mathrm{s}\right.$, $U_{2}=3.78 \mathrm{~m} / \mathrm{s}$ and pressure $=8 \mathrm{bar}$ ), and wavelet transforms at

and internal organisation) are always resolved in relation to the chosen wavelet scale. Thus, if smaller structures have a clumping or clustering recognisable at the chosen wavelet scale, the nature of this organisation is revealed (see e.g. the wavy sheet at $a^{\prime}=0.32$ ). Similarly any sub-structure or organisation within the larger structure, corresponding to the wavelet scale, is revealed (e.g. the details of the rollers, also at $a^{\prime}=0.32$ ). Finally, at each scale, the wavelet captures patterns appropriate to that scale as independent entities. The wavelet transform therefore appears to be a useful tool in the eduction of scale-specific regions of coherence in the flow.

\section{2}

\section{The turbulent mixing layer}

We now turn our attention to the more interesting case of the fully turbulent mixing layer (Fig. 2).

Here too the image was carefully trimmed and the pixels on the upper and lower back-ground of the mixing layer set to a uniform average value corresponding to the respective edge. In the raw image of the turbulent mixing layer, the large-scale structure is again seen to stand out. Clearly these are strikingly similar in gross features to those seen the laminar case. The vortices are clearly seen and grow in size downstream of the splitter plate. The regions of maximum strain (edges of the vortices) are seen as the most intense in the image. In addition,
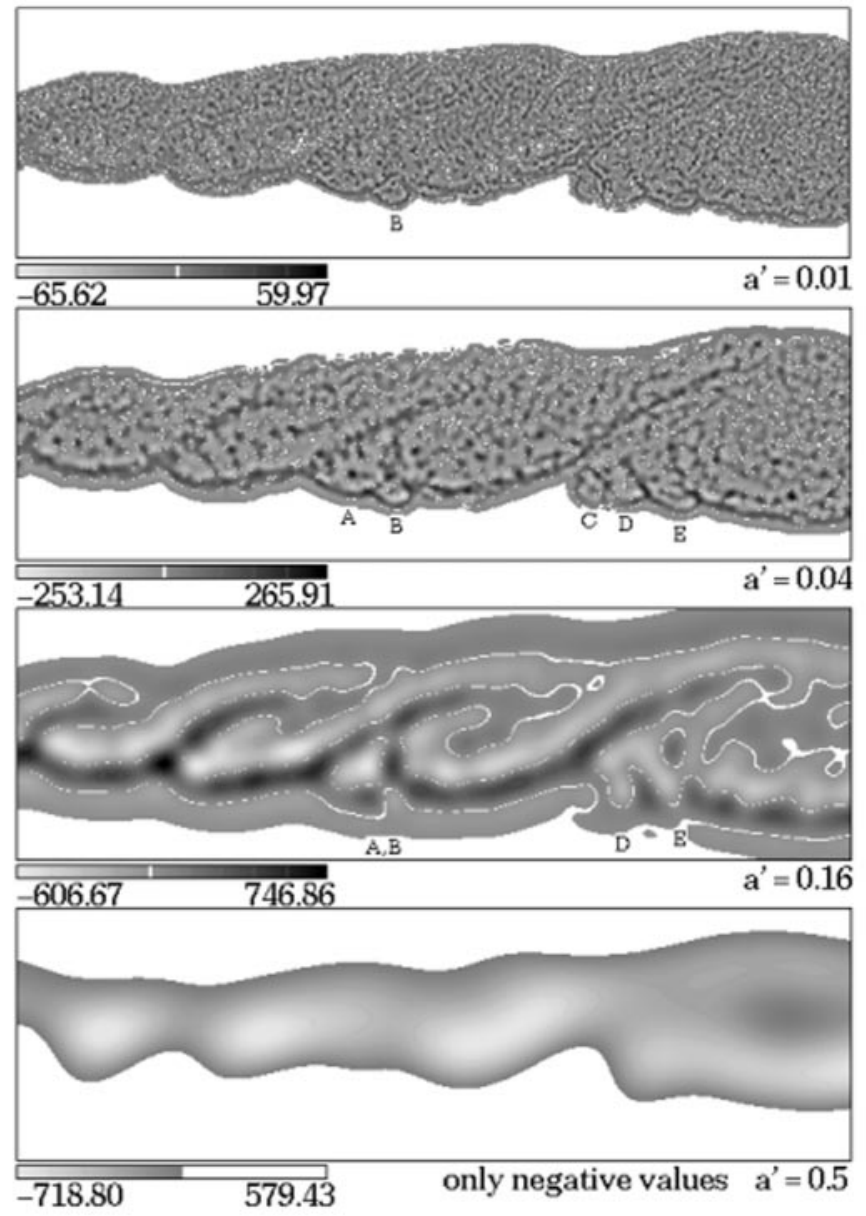

seven different scales

the "bubble-like" regions (marked A, B, C, D, E in Fig. 2) just upstream of each vortex and below the upward rising arm of the strain layer appear as a sub-structure or a series of sub-structures. With the range of physical scales present in the turbulent flow, the scale-specific nature of the wavelet transform becomes a great advantage, and reveals a wide diversity in structural organisation.

Figure 2 also shows the wavelet transforms of the turbulent mixing layer at seven different wavelet scales increasing dyadically from $a^{\prime}=0.01$. It has already been shown above that the wavelet transform picks out organised regions in a flow as isolated structures at the appropriate scales. Thus, perhaps the most surprising result is revealed at the lowest two wavelet scales $\left(a^{\prime}=0.01\right.$ and 0.02$)$. Here the whole mixing layer appears to be covered by a nearly homogeneous distribution of very fine scale structures. These, at first glance, appear to be of approximately the same size, no matter how far downstream one proceeds. (A more quantitative study of this aspect will be reported separately.) On the other hand, Everson et al. (1990), studying dye concentration in longitudinal sections of a jet illuminated by a laser sheet, note that the size of the small-scale structures in the jet increases with downstream distance from the exit nozzle. In striking contrast, the present study suggests that the size of the small-scale structure does not vary significantly with downstream distance $x$ in the mixing layer 
(an observation we shall return to in Sect. 5). Also, while their study (and that of Kailas et al. 1995 for diametral slices of a jet) shows the small scale structure to be "string-like" (crosssections of sheets), it is not so clear in the present study. In fact, the fine structure in the mixing layer appears to be a mixture of broken strings interspersed with beads. A major reason for this observation may well be that in the present case the imagery being analysed is not a section of the flow but (being obtained from a schlieren photograph) represents in some sense a spanwise integral of the flow.

At the smallest two wavelet scales, there appears to be a hint that these structures are more intense (and connected) along the regions of maximum density gradient, e.g. the bottom edge of the mixing layer is clearly more intense and better organized than the top edge. With increasing wavelet scale the connectedness of these regions is more clearly manifested and an intermediate scale structure of the mixing layer becomes apparent. At $a^{\prime}$ greater than 0.04 (and particularly at $a^{\prime}=0.16$ ) the edges of the vortices show clear elongated structures that appear to be cross sections of thick rolled-up sheets. Thus the stringy nature of structures seen in a jet by Everson et al. (1990) and Kailas et al. (1995) at the smallest scales is seen here at scales intermediate between the smallest and the largest. It may be noted, however, that the thickness of the edges does not appear to increase with distance downstream. Again, at larger scales $\left(a^{\prime}=0.08\right)$, though the vortex core is occupied by structures bigger in size than those seen at the smallest scales, these too apparently exhibit the same uniformity of size independent of $x$. Thus, while the overall vortex size grows with downstream distance, the sub-structures do not show growth even if there appears to be elongation of the edges in the $x$-direction.

The "breaking wave" picture is revealed at $a^{\prime}=0.04$ and above; at $a^{\prime}=0.08$ the analogy is particularly strong. The lower arms of the vortices are seen as distinct regions that rise along the edge of a vortex and appear to break up at the top and into the core, much like rollers on a beach.

At $a^{\prime}=0.32$ and 0.5 , the more interesting aspects are revealed by the negative values of the wavelet transform. This is due to the lower over-all intensity of the (larger scale) vortex cores compared to that of the background (see the raw data). The smallest two vortices are seen as distinct entities at these scales. However, the wavelet scale is still too small to recognise the fourth vortex as one single entity, the arms of this vortex being still resolved separately. At $a^{\prime}=0.32$, while the core of the third vortex is seen as a region of high negative intensity (with its extension upwards and downstream between the vortex edges being captured), at $a^{\prime}=0.5$ the third vortex is seen as an isolated entity.

Thus, the analysis reveals structures at three levels. At $a^{\prime}=0.01$ and 0.02 , the whole turbulent mixing layer appears homogeneously "peppered" by fairly uniform small structures. The connectedness of the vortex edges are well defined for $a^{\prime}$ between 0.04 and 0.16 (most clearly at $a^{\prime}=0.16$ ). And the locations of the vortex cores are best seen at the largest scale $\left(a^{\prime}=0.5\right)$. The smallest scales show a mixture of structure shapes, pieces of strings (with no preferred orientation) mixed with beads. The scales in between reveal thick elongated string-like organisation. And those at the largest scale are clearly bead-like.
The manner in which the wavelet transform picks out structures of the appropriate size as the wavelet scale is increased is also revealed in the study of the "bubbles" marked A, B, C, D and E in Fig. 2. The wavelet sees these as distinct entities or sees distinct aspects of these at different scales. At $a^{\prime}=0.02$, bubble B is difficult to distinguish among the fine scale structures as an area enclosing two (or three) smaller structures. These enclosed structures cannot be resolved separately at $a^{\prime}=0.04$ and the bubble is seen as an area enclosing a single structure. At $a^{\prime}=0.08$, it is seen as distinct region of negative intensity with a very thin edge that hardly registers at this scale. At $a^{\prime}=0.16$, the bubble has disappeared as it is too small to be discerned at this scale. Similarly bubble A is barely recognisable upto $a^{\prime}=0.04$, is clearly distinguished at $a^{\prime}=0.08$ and 0.16 as an area of low intensity enclosed by a broken boundary of higher intensity, and finally, at $a^{\prime}=0.32$, it is seen as a single separate entity. The same is the case for bubbles $\mathrm{C}, \mathrm{D}$ and $\mathrm{E}$. This again shows that the wavelet transform is very useful in picking out areas of coherence appropriate to the scale of the wavelet used.

\section{3}

\section{Eduction of structures from wavelet transforms}

A variety of options exist to detect structures from the wavelet-transformed images. In the present case, the best results were obtained by studying the images at various thresholds. The optimum threshold could be determined so that the structures captured revealed details unique to that scale but ignored unnecessary clutter.

Figure 3 shows the structures so revealed for the laminar mixing layer at different wavelet scales at the thresholds shown. Several interesting features stand out. At $a^{\prime}=0.02$, nearly every roll-up of each vortex is clearly revealed. At the next larger scale, the details of the smallest two vortices are no longer clear. At $a^{\prime}=0.08$, the internal rolled-up layers of the fourth, fifth and sixth vortices are broken up (the breaking roller analogy). At $a^{\prime}=0.16$, the cores of the fifth and sixth vortices are clearly marked out. At $a^{\prime}=0.32$, at the threshold chosen, the first four vortices merge into a thick wavy sheet while the fifth and sixth vortices are seen to be like breaking rollers. At the highest scale $a^{\prime}=0.5$, the fifth and sixth vortices are picked up as separate isolated entities. However, while each wavelet scale reveals a distinct feature of the laminar mixing layer, the relationships between one scale and the next and the raw image is clearly discernible.

Figure 4 shows the structures determined from the wavelet transforms of the turbulent mixing layer, again at the thresholds shown. At the lowest wavelet scales $\left(a^{\prime}=0.01,0.02\right.$ and even 0.04), the homogeneous distribution of the small scale structure is very clearly seen. Konrad (1976) has studied the density variation within the mixing layer, and shown that there remain small blobs of non-turbulent fluid ingested by the large vortices during growth but remaining unmixed within the mixing layer. Also, Roshko (1976) had concluded that the significant intermittency (with rapid fluctuations between values corresponding to the pure fluid on either side of the mixing layer), seen in point measurements of passive transportable scalars in a mixing layer, indicated the presence of distinctly separate small-scale structure embedded within the large-scale vortices. The wavelet transform pictures do 



Fig. 3. Structures educed from the wavelet transform of the laminar mixing layer

show a significant small scale structure in the fully turbulent mixing layer consistent with the above picture. But, additionally, they reveal that these are relatively homogeneously distributed, so that the small blobs of fluid appear to be spread rather evenly throughout the mixing layer, again nearly independently of distance downstream. The large scale organisation is very difficult to discern in the wavelet transform pictures at these scales. Thus, unlike in the laminar case, the small scale structure is significantly uncorrelated to the large scale structure or the raw image. It may be worth remembering here that Bernal and Roshko (1986) had shown that the onset of three-dimensionality in the small-scale motion does not destroy the spanwise coherence of the large scale motion.

Although some hints of organisation along the regions of strain (the arms of the vortices) are revealed at the smallest scales, this becomes more clearly apparent only at the intermediate scales $a^{\prime}=0.08,0.16$ (and to some extent for the largest vortex even at $a^{\prime}=0.32$ and 0.5 ). The structure of the turbulent mixing layer at these scales shows similarities to that of the laminar case. The above is consistent with Roshko (1976, his Fig. 10), who has shown how the edges of the mixing layer are more "unmixed" than the centres. Also, Wygnanski et al. (1979) showed that the cores have much shorter spin-wise characteristic lengths than the edges of the mixing layer. Again, the thickness of the vortex boundaries appears to remain constant with downstream distance. This invariance is also seen in the case of the internal sub-structures inside each vortex, upto $a^{\prime}=0.08$, the sub-structures at higher wavelet

scales in the smaller vortices apparently being too weak in intensity and organisation relative to the vortex edges to be resolved at the thresholds chosen.

Interestingly, the above also shows that the wavelet picks out all types of structures at a given wavelet scale, even if these are of widely varying aspect ratios - such as the highly elongated arms and the smaller more rounded internal sub-structures. It may therefore be concluded that the nature and distribution of such scale-specific structures are aspects of the flow organisation and not artifacts of a particular wavelet scale.

The similarity of vortices to breaking rollers is clearly seen at $a^{\prime}=0.08$ (and at 0.16 , for the third and fourth vortex). At $a^{\prime}=0.5$, the location, shape and size of the first three vortex cores are clearly seen, while the wavelet is still too small to "see" the fourth vortex as a separate entity, its lower arm being picked up clearly. Interestingly, the elongation of the vortex cores in the downstream direction as seen in the raw image is clearly captured.

The manner in which aspects of the bubble sub-structures appear at differing scales is also revealed. Thus bubble A is seen at $a^{\prime}=0.01$ and 0.02 as an organisation of fine structures along a barely discernible boundary, with other fine structures (of apparently the same size, but weaker organisation or connectivity) embedded within. At $a^{\prime}=0.04$ and 0.08 , the boundaries of the bubble are clearly defined but only the most intense internal region is picked up at $a^{\prime}=0.08$. At $a^{\prime}=0.16$, only the more intense and connected boundary regions are picked up, the internal structures being too weak in intensity 

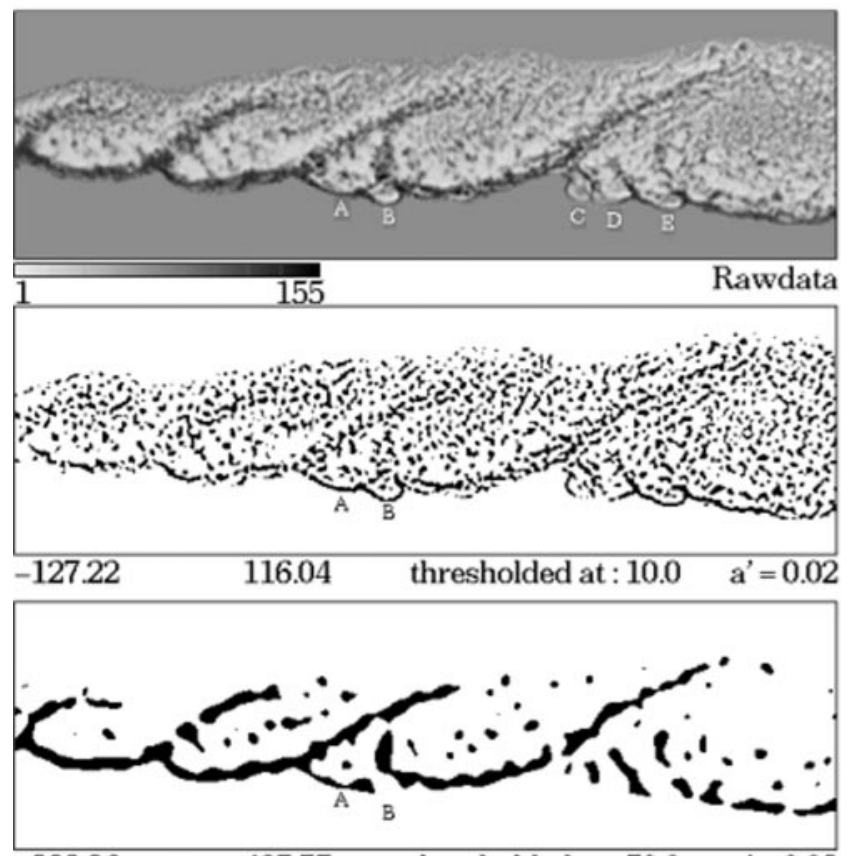

$\begin{array}{llll}-389.36 & 425.55 & \text { thresholded at : } 50.0 & \mathrm{a}^{\prime}=0.08\end{array}$

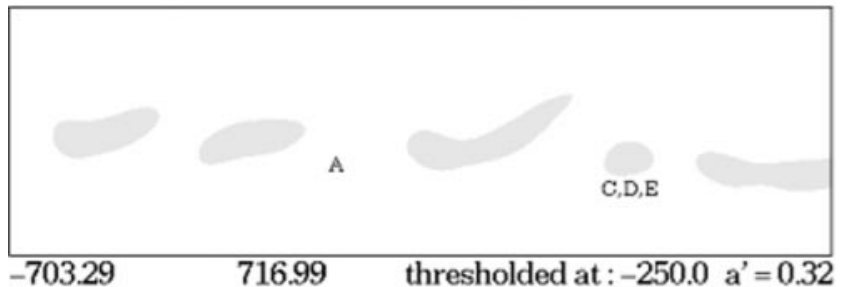

Fig. 4. Structures educed from the wavelet transform of the turbulent mixing layer

and too loosely organized to be revealed. At $a^{\prime}=0.32$, the whole bubble is seen as an isolated structure, but it is not seen at $a^{\prime}=0.5$, the wavelet at this scale being too large to resolve structures of this size. A similar sequential detection, with increasing wavelet scale, of different aspects is seen for bubbles $\mathrm{C}, \mathrm{D}$ and $\mathrm{E}$, culminating in the whole set of bubbles being recognised as a single coherent entity at $a^{\prime}=0.32$.

At all scales, the larger vortices, further away from the splitter plate, enclose more small structures than the smaller vortices closer to the splitter plate, this being especially clear at $a^{\prime}=0.08$ and 0.16 . Roshko (1976) states that small scale turbulence mixing is important at high Reynolds numbers to account for the decrease of vorticity as the mixing layer grows by ingestion of irrotational fluid. The presence of more small scale regions enclosed within the vortices further away from the splitter plate (see $a^{\prime}=0.08$ and 0.16 ) supports this view.

In summary, unlike the laminar mixing layer, three levels of scale-specific structures are revealed in the turbulent mixing layer: the smallest scale homogeneous structure, the intermediate scale organisation of the vortex boundaries (and some internal sub-structures), and the large-scale coherence of each vortex itself. While the latter two structure types are discernible in the raw image (and in the laminar case), the smallest scale structures reveal an organisation very difficult to discern
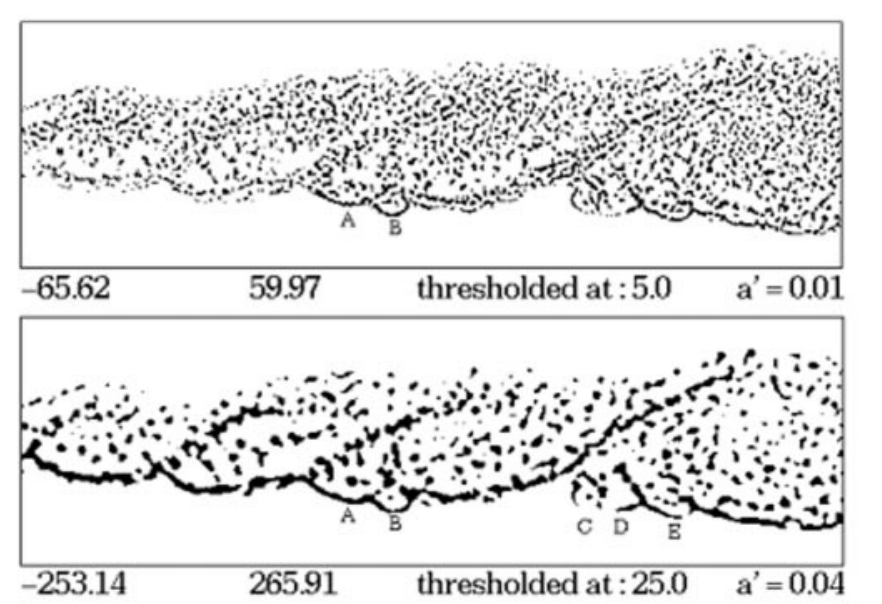
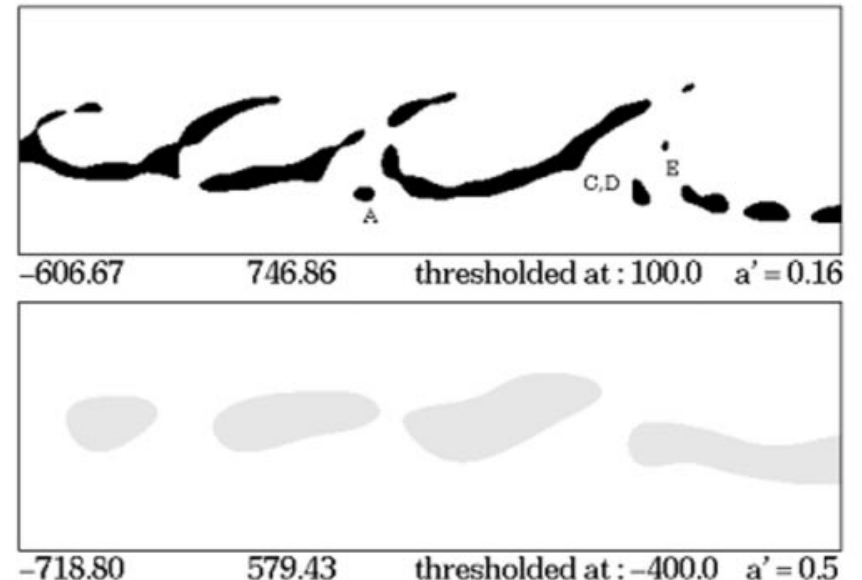

from the raw image, and appear relatively uncorrelated to the large scale organisation; they are certainly not seen in the laminar case. This again is unlike the situation seen in a round turbulent jet either in longitudinal sections (Everson et al. 1990) or diametral ones (Kailas et al. 1995), where the structures detected bear organisational semblance across wavelet scales and to the raw image.

\section{5}

\section{Conclusions}

The present work shows that, in the laminar case, the small-scale structure is similar to what is seen in the raw images itself, the wavelet transform tracing out the organisation of the sharp density gradients more clearly. So also the large scale structure, the vortices being detected as single coherent structures at the appropriate wavelet scale. However, in the turbulent mixing layer, while the large-scale wavelet picture reveals clearly the coherence visible in the raw data, the small scale picture bears only a weak correlation to the large scale picture, unlike the case of jet imagery (Everson et al. 1990; Kailas et al. 1995). It is here that the value of the wavelet transform is best exhibited in this flow: the small structures, difficult to discern in the raw data, are more clearly revealed. Furthermore, these show noticeable homogeneity in distribution and uniformity in size with downstream distance. This is 
in sharp contrast to current views about the small scale structure of other free shear flows such as jets (Everson et al. 1990). The explanation for this probably lies in the dependence of the Kolmogorov length scale ( say $\ell_{*}$ ) on downstream distance in different flows. It is well known that $\ell_{*} \sim \varepsilon^{-1 / 4}$ where $\varepsilon$ is the specific dissipation, proportional to $U^{3} / \delta$ where $U$ and $\delta$ are characteristic large-eddy velocity and length scales. Using the standard similarity laws, it is easily seen that $\ell * \sim x^{1 / 4}$ in a mixing layer and $\ell * \sim x$ in a round jet: the small scales are much less sensitive to downstream distance in a mixing layer compared to a jet. It is remarkable that the low-scale wavelets are able to detect this feature of the small scale motion in the flow.

Besides the above, there appear to be two other levels of structure organisation in the turbulent mixing layer. These are discernible in the raw image itself and are similar to those seen in the laminar case. Thus, the edges of the large structures stand out as more intense and better organised contiguous regions than the vortex centres at scales in between the smallest and largest. This is in agreement with the known fact that these edges are more highly correlated in the spanwise direction than are the cores (Roshko 1976). At these scales, the wavelet transform picture clearly brings out the often stated analogy of the mixing layer being akin to breaking rollers. Finally, each vortex is seen as a single coherent entity at the largest scales.

In interpreting the present pictures, it must of course be remembered that they represent spanwise integrals over the flow because of the optical technique used. We see greater potential for analysis of visualizations of sections of the flow, e.g. by laser-sheet illumination with fluorescent dye, and will publish separately results on jet flows. The objective of the present work has been to show that the wavelet transform can provide a powerful tool for inferring structure from instantaneous data.

\section{References}

Bernal LP; Roshko A (1986) Streamwise vortex structure in plane mixing layer. J Fluid Mech 170: 499-525

Bernal LP (1988) The statistics of the organised vortical structure in turbulent mixing layers. Phys Fluids 31: 2533-2543

Brown GL; Roshko A (1974) On density effects and large structures in turbulent mixing layers. J Fluid Mech 64: 775-816

Cutler AD; Johnson CH (1997) Analysis of intermittency and probe data in supersonic flow with injection. Exp Fluids 23: 38-47

Everson R; Sirovich L; Sreenivasan KR (1990) Wavelet analysis of the turbulent jet. Phys Lett A 145: 314-322

Farge M (1992) Wavelet transforms and their applications to turbulence. Ann Rev Fluid Mech 24: 395-457

Fiedler HE (1988) Coherent structures in turbulent flows. Progr Aerospace Sci 25: 231-270

Hussain AKMF (1986) Coherent structures and turbulence. J Fluid Mech 173: 303-356

Kailas SV; Bhat GS; Kalavathi K (1995) On coherent structures in a round turbulent jet educed by wavelet transforms. Proc. 6th Asian Cong. Fluid Mech., Singapore, May 1995, pp. 993-997

Kline SJ; Reynolds WC; Schraub FA; Runstadler PW (1967) Structure of turbulent boundary layers. J Fluid Mech 30: 741-773

Konrad JH (1976) An experimental investigation of mixing in turbulent shear layer and wakes with applications to diffusion limited chemical reactions. Ph.D. Thesis, California Inst. of Tech. Pasadena. California

Marr D (1982) Vision. New York: Freeman and Co
Meyer Y (1993) Wavelets: algorithms and applications. Philadelphia: SIAM

Robinson SK (1991) Coherent motions in the turbulent boundary layer. Ann Rev Fluid Mech 23: 601-639

Roshko A (1976) Structure of turbulent shear flows: a new look. AIAA J 44: $1349-1357$

Strang G (1989) Wavelets and dilation equation: a brief introduction. SIAM Rev 31: 614-627

Tso J; Hussain F (1989) Organised motions in fully developed turbulent axisymmetric jet. J Fluid Mech 203: 425-448

Wygnanski I; Oster D; Fiedler H; Dziomba B (1979) On the perseverance of a quasi-two-dimensional eddy-structure in a turbulent mixing layer. J Fluid Mech 93: 325-335 